\title{
Mécanismes physiopathologiques des infections digestives à Clostridium difficile
}

Clostridium difficile est l'agent pathogène spécifique de la colite pseudo-membraneuse et est responsable de $25 \%$ des diarrhées observées au cours ou au décours d'une antibiothérapie. Les souches toxinogènes de cette bactérie anaérobie produisent deux toxines thermolabiles et cytotoxiques : une toxine $A$ (ou enterotoxine) et une toxine $B$ (ou cytotoxine). Après rupture de l'effet barrière naturel du tube digestif, favorisée par les antibiotiques, et prolifération de $C$. difficile, la toxine $A$ se fixerait à un récepteur glycoprotéique spécifique de la bordure en brosse, entraînant un afflux de polynucléaires au niveau de la lamina propria et une intense réaction inflammatoire. La toxine B, qui est dépourvue d'effet direct sur l'épithélium intestinal, pourrait alors exercer sa forte activité cytotoxique en synergie avec la toxine A sur la muqueuse digestive préalablement lésée par cette dernière.

\section{Frédéric Barbut Gérard Corthier Jean-Claude Petit}

\section{ADRESSE}

F. Barbut : stagiaire doctoral, attaché des hôpitaux de Paris.

J.-C. Petit : docteur ès sciences, professeur des universités, praticien hospitalier. Service de bactériologie-virologie, hôpital Saint-Antoine, 75012 Paris, France.

G. Corthier : docteur ès sciences, directeur de recherche Inra. LEPSD, Inra, 78352 Jouy-en-

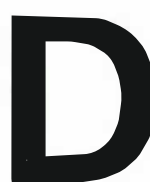

epuis leur apparition en thérapeutique, les antibiotiques ont toujours été incriminés dans la survenue de certaines diarrhées iatrogènes de gravité variable. A partir des années 1950, les tétracyclines et le chloramphénicol ont été rendus responsables d'une atteinte très particulière de la muqueuse rectocolique : la colite pseudo-membraneuse (CPM), dont les cas se sont mutipliés dans les années 1970 avec l'introduction de la lincomycine et de la clindamycine. Si le déséquilibre de la flore intestinale induite par l'antibiothérapie a longtemps été le seul mécanisme d'action retenu pour expliquer l'origine de ces diarrhées, la découverte par Bartlett, en 1978 , de Clostridium difficile dans les selles de patients atteints de CPM a suscité un réel intérêt pour cette bactérie anaérobie peu connue alors des bactériologistes [1]. Dès lors, de nombreuses observations, à la fois in vivo et in vitro, ont permis de mettre en évidence le rôle majeur de deux toxines produites par $C$. difficile: une toxine A (entérotoxine) et une toxine B (cytotoxine) [2]. A l'heure actuelle, bien que des progrès décisifs aient été accomplis dans la connaissance de ces toxines, leur mécanisme d'action exact reste entaché de zones d'ombre.

\section{Un spectre} clinique étendu

La responsabilité de $C$. difficile ne fait aucun doute dans la survenue des $m / s n^{\circ} 3$, vol. 8 , mars 92 
CPM associées à une antibiothérapie où il est retrouvé dans plus de $90 \%$ des cas [3]. Bien que presque tous les antibiotiques (excepté les aminosides administrés par voie parentérale) aient pu être incriminés dans la survenue d'une telle affection, certains d'entre eux sont plus fréquemment en cause: céphalosporines $(30$ à $60 \%$ de cas), aminopénicillines (30 à $40 \%$ des cas), lincosamides (15 à $20 \%$ des cas). Ces proportions sont avant tout le 'reflet du degré de la prescription médicale de ces antibiotiques; le risque d'apparition de diarrhées à $C$. difficile encouru pour chacun d'eux est variable ; ainsi, si l'on prend pour référence la pénicilline à spectre étroit, le risque est multiplié par 8 avec les pénicillines résistantes aux bêtalactamases, par 40 avec les céphalosporines et par 70 avec les lincosamides (cité dans [4]). L'expression clinique des CPM débute brutalement par une diarrhée liquide, abondante, faite de selles crémeuses ou verdâtres, hétérogènes (aspect "grumeleux"), riche en débris muqueux et en général non sanglantes; elle survient de 4 à 10 jours après le début de l'antibiothérapie, voire après l'arrêt du traitement. Elle s'accompagne souvent de fièvre (75\% des cas) et de douleurs abdominales. Les signes biologiques ne sont pas spécifiques: hyperleucocytose, déshydratation extracellulaire et présence d'une entéropathie exsudative avec déperdition protéique. Compte tenu de la gravité des complications (mégacôlon toxique, perforation intestinale), le diagnostic doit être porté en urgence ; il repose sur l'exploration endoscopique permettant la visualisation des fausses membranes. Price et Davis [5] distinguent trois stades d'évolution dont le deuxième représente la forme la plus typique et bien connue des CPM : les lésions siègent le plus souvent au niveau du côlon mais peuvent également se retrouver au niveau du rectum ou plus exceptionnellement du grêle. On peut observer des taches jaunâtres aphtoïdes, arrondies, de $2 \mathrm{~mm}$ de diamètre, régulières, disséminées sur une muqueuse saine ou congestive. L'examen histologique met en évidence une nécrose de la muqueuse colique associée à un exsudat constitué de fibrine, de polynu- cléaires, de débris cellulaires et de mucus. Les examens anatomopathologiques permettent d'effectuer un diagnostic différentiel avec la maladie de Crohn, la rectocolite hémorragique ou les colites à cytomégalovirus.

L'intérêt porté à $C$. difficile a permis d'enrichir le spectre clinique des infections digestives dues à ce germe. Si sa responsabilité est prouvée dans les CPM post-antibiotiques, elle demeure probable dans $30 \%$ des cas de CPM primitives et $25 \%$ des cas de diarrhées consécutives aux antibiotiques mais sans CPM [3]. Le rôle de C. difficile a aussi été évoqué dans les cas de colites aiguës associées aux agranulocytoses, dans les entérocolites associées à la maladie de Hirschsprung (mégacôlon congénital), dans certaines poussées de la maladie de Crohn, mais sa responsabilité demeure encore du domaine de l'hypothétique.

Il existe un portage asymptomatique de $C$. difficile : celui-ci est particulièrement important chez le nouveau-né et le nourrisson, puisqu'il peut être observé chez 20 à $50 \%$ d'entre eux. Malgré la présence de ce germe et de ses toxines au niveau intestinal, les nouveau-nés ne présentent, le plus souvent, aucun signe clinique ni de lésion colique [6]. Le portage asymptomatique est estimé à $3 \%$ chez l'adulte sain, mais la détection de la cytotoxine dans les selles est, dans ce cas, exceptionnelle $(0,3 \%)$. La présence de $C$. difficile chez l'adulte sain est cependant bien supérieure en milieu hospitalier où de récentes observations ont montré la transmission nosocomiale de ce germe: en effet, celui-ci a été retrouvé sur les mains de $59 \%$ du personnel hospitalier au contact de malades infectés [7].

\section{Études \\ physico-chimiques des toxines $A$ et $B$}

C. difficile est une bactérie anaérobie stricte, sporulée, dont plus de $95 \%$ des souches isolées de selles diarrhéiques produisent simultanément et en

\begin{tabular}{|c|c|c|}
\hline \multicolumn{3}{|c|}{$\begin{array}{l}\text { Tableau I } \\
\text { PHYSICO-CHIMIQUES } \\
\text { B DE CLOSTRIDIUM DIFFICILE }\end{array}$} \\
\hline Propriétés & Toxine A & Toxine B \\
\hline $\begin{array}{l}\text { Poids moléculaire (kDa) } \\
\text { PAGE } \\
\text { PAGE-SDS }\end{array}$ & $\begin{array}{c}400 \text { à } 600 \\
308\end{array}$ & $\begin{array}{l}360 \text { à } 500 \\
280 \text { à } 300\end{array}$ \\
\hline Point isoélectrique & 5,5 & 4,1 à 4,4 \\
\hline $\begin{array}{l}\text { Composition en } \\
\text { acides aminés (aa) }\end{array}$ & \multicolumn{2}{|c|}{$\begin{array}{l}\text { Les toxines } A \text { et } B \text { sont riches en Asp, Glu et } \\
\text { Gly, et pauvres en Hist et aa soufrés }\end{array}$} \\
\hline $\begin{array}{l}\text { Effet de la } \\
\text { température }\end{array}$ & Inactivation à $56^{\circ} \mathrm{C}$ & Inactivation à $56^{\circ} \mathrm{C}$ \\
\hline $\begin{array}{rll}\text { Stabilité à pH } & 2 \\
\mathrm{pH} & 10\end{array}$ & $\begin{array}{l}+ \\
-\end{array}$ & $\begin{array}{l}- \\
-\end{array}$ \\
\hline $\begin{array}{l}\text { Inactivation par: } \\
\text { trypsine } \\
\text { chymotrypsine } \\
\text { pronase } \\
\text { oxydation } \\
\text { détergents } \\
\text { agents réducteurs }\end{array}$ & $\begin{array}{l}\text { non } \\
\text { oui } \\
\text { oui } \\
\text { oui } \\
\text { oui } \\
\text { non }\end{array}$ & $\begin{array}{l}\text { oui } \\
\text { oui } \\
\text { oui } \\
\text { oui } \\
\text { oui } \\
\text { non }\end{array}$ \\
\hline
\end{tabular}

(D'après [2].)

PAGE : électrophorèse en gel de polyacrylamide; PAGE-SDS : électrophorèse en gel de polyacrylamide dans des conditions dénaturantes (SDS : dodécylsulfate de sodium). 


\section{RÉFÉRENCES}

1. Bartlett JG, Moon N, Chang TW, Taylor N, Onderdonk AB. Role of Clostridium difficile in antibiotic associated pseudomembranous colitis. Gastroenterology 1978 ; $75: 778-82$.

2. Lyerly DM, Krivan HC, Wilkins TD. Clostridium difficile: its disease and toxins. Clin Microbiol Rev 1988; 1 : 1-18.

3. Matuchansky C. Spectre clinique des infections intestinales à Clostridium difficile. In : Rambaud JC, Ducluzeau R, eds. Clostridium difficile et pathologie intestinale. Paris : Springer-Verlag, $1990: 17-23$.

4. Marteau P, Sobhani I, Berretta O, Rambaud JC. Physiopathologie des infections intestinales dues à Clostridium difficile. Rôle de l'écosystème colique. Gastroentérol Clin Biol $1991 ; 15: 322-9$.

5. Price AB, Davies DR. Pseudomembranous colitis. J Clin Pathol 1977; 30 : 1-12.

6. Stuphen JL, Grang RJ, Flores A, Chang TW, Bartle HJG. Chronic diarrhoea associated with Clostridium difficile in children. $\mathrm{Am}$ $J$ Dis Chil 1983 ; 137 : 275-8.

7. McFarland LV, Surawicz CM, Stamm WE. Risk factors for Clostridium difficile carriage and $C$. difficile associated diarrhea in a cohort of hospitalized patients. $J$ Infect Dis $1990 ; 162: 678-84$

8. Wilkins TD, Lyerly DM. Toxins of Clostridium difficile and Clostridium sordelii. In : Rambaud JC, Ducluzeau R, eds. Clostridium difficile et pathologie intestinale. Paris Springer-Verlag, 1990 : 45-53.

9. Dove $\mathrm{CH}$, Wang SZ, Price SB, et al. Molecular characterization of the Clostridium difficile toxin A gene. Infect Immun 1990 ; $58: 480-8$.

10. Phelps CJ, Lyerly DL, Johnson JL, Wilkins TD. Construction and expression of the complete Clostridium difficile toxin A gene in Escherichia coli. Infect Immun 1991; 59 : 150-3.

11. Krivan HC, Clark GF, Smith DF, Wilkins TD. Cell surface binding site for Clostridium difficile enterotoxin : evidence for a glycoconjugate containing the sequence Gal $\alpha 1-3 \mathrm{Gal} \beta 1-4 \mathrm{G} 1 \mathrm{cNAc}$. Infect Immun 1986 ; $53: 573-81$

12. Clark GF, Krivan HC, Wilkins TD, Smith DF. Toxin A from Clostridium difficile binds to rabbit erythrocyte glycolipids with terminal Gal $\alpha 1-3 \mathrm{Gal} \beta 1-4 \mathrm{G} 1 \mathrm{cNAc}$ sequences. Arch Biochem Biophys 1987; 257 : quantité variable des toxines $\mathrm{A}$ et $\mathrm{B}$. Ces deux toxines, impliquées dans la physiopathologie des CPM et diarrhées post-antibiotiques, agissent au niveau de l'entérocyte. Leur purification et leur caractérisation physicochimique ont été deux étapes décisives dans la compréhension de leur mode d'action.

La purification s'effectue à partir du surnageant de culture d'un bouillon cœur-cervelle par différentes méthodes chromatographiques (échange d'ions, gel filtration, affinité). Si la caractérisation physico-chimique de ces toxines s'est longtemps heurtée à la présence de protéines contaminantes éluées en même temps que la toxine $\mathrm{A}$, les méthodes plus récentes fondées sur l'affinité vis-à-vis de certaines glycoprotéines (comme la thyroglobuline) ont permis d'éviter cet écueil [8].

Les deux toxines sont remarquables par leur grande taille: selon les auteurs, leur poids moléculaire est évalué en électrophorèse sur gel de polyacrylamide et dans des conditions dénaturantes, entre 250 et $300 \mathrm{kDa}$. Ces toxines sont des protéines (inactivation par la chymotrypsine et la pronase), non dissociables en sousunités et riches en acide aspartique, acide glutamique et glycine. Elles sont toutes les deux inactivées à $56{ }^{\circ} \mathrm{C}$ (Tableau I).

Le gène codant pour la toxine $\mathrm{A}$ a récemment été cloné et séquencé $[9$, 10] ; il se compose de 8133 pb qui codent pour un polypeptide actif de 308 kDa formé de 2710 acides aminés. Un tiers du gène à l'extrémité 3' est constitué de 38 séquences répé- titives qui sont regroupées en deux classes sur la base de leur taille et de leur homologie en nucléotides (figure 1). Cette portion du génome qui code pour la partie C-terminale de la toxine a été séparément clonée et exprimée dans Escherichia coli; le peptide qui en résulte se fixe, tout comme la toxine native, à une glycoprotéine contenant un motif trisaccharidique peu commun (Gal $\alpha 1-3 \mathrm{Gal} \beta 1-4 \mathrm{G} 1 \mathrm{cNAc})$ présent au niveau des hématies de lapin qu'il est capable d'agglutiner, et de la membrane de la bordure en brosse du lapin et du hamster (figure 2) [11, 12]. En étudiant la fixation de la toxine A purifiée et marquée au tritium aux récepteurs iléaux de la bordure en brosse de lapin, Pothoulakis et al. [13] ont récemment montré que ces derniers sont couplés à une protéine $G$ membranaire. Les domaines répétitifs de la partie C-terminale de la toxine A pourraient se lier à plusieurs motifs hydrocarbonés permettant sans doute une stabilisation de la toxine sur la membrane. Par conséquent, le trisaccharide Gal $\alpha 1-3 \mathrm{Gal} \beta 1-4 \mathrm{G} 1 \mathrm{cNAc}$ semble jouer le rôle d'un véritable récepteur fonctionnel de la toxine $\mathrm{A}$; d'ailleurs, les lignées cellulaires qui expriment à leur surface ce motif (comme par exemple les lignées F9, ou OTF9-63, ou P19) sont beaucoup plus sensibles aux effets cytopathiques de la toxine A que les autres lignées. Situé en amont du gène de la toxine $A$ se trouve celui de la toxine $\mathrm{B}$, dont le clonage et le séquençage ont été réalisés par Barroso et al. [14] et von Eichel-Steiber et al. [15]. La proximité de ces deux

\begin{tabular}{|l|l|l|l|}
\hline $5^{\prime}>7 \mathrm{~kb}$ & $0,4 \mathrm{~kb}$ & \multicolumn{2}{c|}{$8,1 \mathrm{~kb}$} \\
\hline & & & \\
\hline & $17 \mathrm{kDa}$ & \multicolumn{2}{c|}{ Séquences répétitives } \\
Toxine B & Toxine A
\end{tabular}

Figure 1. Représentation schématique des gènes des toxines $A$ et $B$ de Clostridium difficile. Entre les deux gènes codant pour les toxines $A$ et $B$ (qui sont des protéines de 250 à $300 \mathrm{kDa}$ ), on trouve une petite séquence ouverte ayant le potentiel de coder pour un polypeptide de $17 \mathrm{kDa}$. 


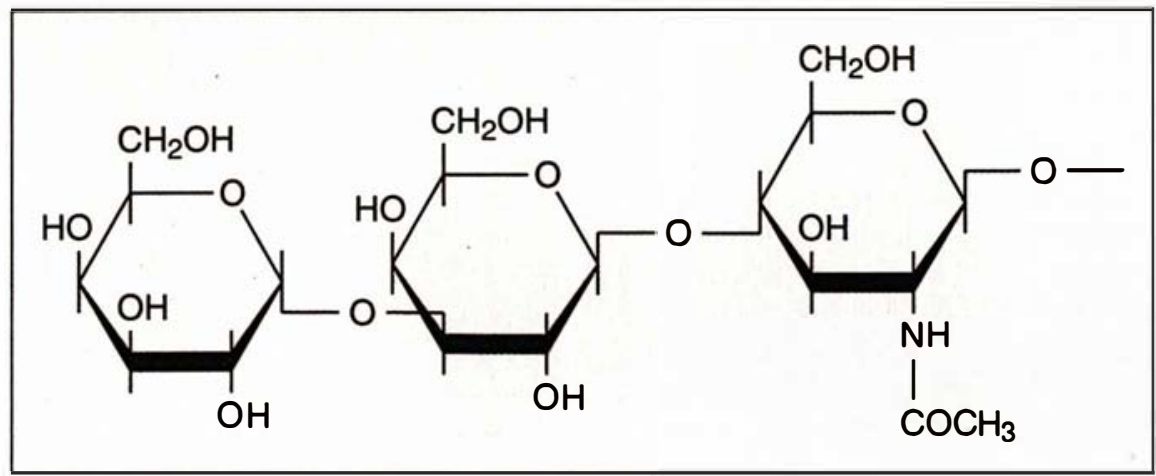

Figure 2. Structure du motif trisaccharidique auquel se fixe la toxine A au niveau de la bordure en brosse. la sécrétion des toxines et une meilleure compréhension de la physiopathologie des infections intestinales dues à ce germe.

\section{Mécanismes d'action des toxines}

Actuellement, la connaissance des mécanismes d'action des toxines de $C$. difficile repose sur des observations in vivo (reproduction d'une CPM expérimentale chez le hamster, modèle de l'anse iléale ligaturée de lapin, études expérimentales de l'infection chez des souris axéniques*) et in vitro (effets des toxines sur différentes lignées cellulaires). Grâce à des approches différentes et complémentaires tant sur les plans écologique, histologique que biochimique, les mécanismes physiopathologiques des infections digestives à $C$. difficile sont aujourd'hui mieux compris. Longtemps, les études ont porté sur la toxine A considérée alors comme le principal facteur de pathogénicité ; à l'heure actuelle, la plupart des auteurs s'accordent à penser que les deux toxines agissent en synergie et qu'elles sont, toutes deux, indispensables à l'expression du pouvoir pathogène de $C$. difficile.

- Les modèles in vivo

- CPM expérimentale du hamster

L'étiologie et le traitement des CPM post-antibiotiques ont largement bénéficié des travaux effectués sur des modèles animaux comme la souris conventionnelle, le cobaye, le hamster, le chien de prairie, le lapin ou le lièvre chez lesquels la maladie peut être reproduite expérimentalement [18]. Ainsi l'administration per os de clindamycine au hamster doré de Syrie - modèle animal le plus largement répandu - entraîne l'apparition d'une diarrhée en 3 à 7 jours et la mort de l'animal dans les 24 heures qui suivent le début des premiers symptômes. Les selles du hamster malade contiennent alors de grandes quantités de $C$. difficile ainsi que de ses toxines. L'examen histologique des biopsies digestives révèle une réaction inflammatoire et une - Un animal axénique est un animal dépourou de
germe. 


\section{RÉFÉRENCES}

13. Pothoulakis C, LaMont JT, Eglow R, Gao N, Rubins JB. Characterization of rabbit ileal receptors for Clostridium difficile toxin A. J Clin Invest 1991; 88 : 119-25.

14. Barroso LA, Wang SZ, Phelps CJ, Johnson JL, Wilkins TD. Nucleotide sequence of Clostridium difficile toxin B gene. Nucleic Acids Res 1990 ; 18 : 4004

15. Von Eichel-Steiber C, LaufenbergFcldmann R, Sartingen S, Schulze J, Sauerborn M. Cloning of Clostridium difficile toxin $\mathrm{B}$ gene and demonstration of high $\mathrm{N}$ terminal homology between toxin A and B. Med Microbiol Immunol Berl 1990; 179 : 271-9.

16. Kato $\mathrm{N}, \mathrm{Ou} \mathrm{CY}$, Kato $\mathrm{H}$, et al. Identification of toxigenic Clostridium difficile by the polymerase chain reaction. I Clin Microbiol 1991 ; 29 : 33-7.

17. Wren B, Clayton C, Tabaqchali S Rapid identification of toxigenic Clostridium difficile by polymerase chain reaction. Lan cet $1991 ; 335: 423$.

18. Elmer GW. Animals models of human Clostridium difficile infections. In : Rambaud JC, Ducluzeau R, eds. Clostridium difficile et pathologie instestinale. Paris : SpringerVerlag, 1990: 25-36.

19. Rolfe RD. Binding kinetics of Clostri dium difficile toxins $\mathrm{A}$ and $\mathrm{B}$ to intestina brush border membranes from infant and adult hamsters. Infect Immun 1991; 59 1223-30.

20. Toothaker RD, Elmer GW. Prevention clindamycin-induced mortality in hamsters by Saccharomyces boulardii. Antimicrob Agents Chemother 1984; 26 : 552-6.

21. Surawicz CM, MacFarland LV, Elmer G, Chin MD. Treatment of recurrent Clos tridium difficile colitis with vancomycine and Saccharomyces boulardii. Am J Gastroenterolog) 1989 ; 84 : 1285-7.

22. Torres J, Jennische E, Lange S, Lönnroth I. Clostridium difficile toxin A induces a specific antisecretory factor which protects against intestinal mucosal damage. Gut $1991 ; 32: 791-5$

23. Czuprynski CJ, Johnson WJ, Balish E, Wilkins T. Pseudomembranous colitis in Clostridium difficile monoassociated rats. Infect Immun 1983 ; 39 : 1368-76. nécrose du cæcum, parfois de l'iléon distal, voire du côlon. En revanche, les fausses membranes sont rarement mises en évidence, à la différence des CPM chez l'homme. Il est clairement établi, à l'heure actuelle, que l'administration d'antibiotiques entraîne une rupture de l'effet barrière naturel de la flore du tube digestif, créant ainsi un contexte favorable à l'implantation et à la prolifération des souches de $C$. difficile toxinogènes, qu'elles soient d'origine endogène ou acquises dans l'environnement.

Lyerly et al. [2] ont montré que l'administration orale de 5 à $10 \mu \mathrm{g}$ de toxine A purifiée ou d'un filtrat de culture de $C$. difficile au hamster reproduit les mêmes symptômes. En revanche, la toxine $B$ administrée en intragastrique n'exerce aucun effet. Cependant elle devient létale lorsqu'elle est associée à de très faibles quantités (sublétales) de toxine A ou lorsque le cæcum a été mécaniquement lésé auparavant. Ainsi, si la toxine $\mathrm{A}$ a longtemps été reconnue comme le principal facteur de pathogénicité de la CPM, la toxine B revêt aussi un rôle important nécessitant la présence de la toxine A. C'est d'ailleurs pourquoi. le hamster doit être immunisé à la fois contre les toxines $\mathrm{A}$ et $\mathrm{B}$ pour être totalement protégé de la CPM induite par clindamycine.

Un fait plus intéressant est de constater que les hamsters nouveau-nés tout comme les nouveau-nés chez l'homme - sont réfractaires à la CPM bien qu'hébergeant au niveau intestinal un grand nombre de souches toxinogènes de $C$. difficile et une importante quantité de toxines. Récemment, Rolfe et al. [19] ont étudié les cinétiques de fixation des toxines $\mathrm{A}$ et $\mathrm{B}$ aux membranes des bordures en brosse des hamsters adultes et nouveau-nés ; ils ont montré clairement qu'il n'existe aucune différence significative dans les paramètres cinétiques de fixation de la toxine $\mathrm{A}$ et/ou B susceptible d'expliquer les différences, liées à l'âge de sensibilités aux maladies digestives induites par C. difficile.

Les modèles animaux sont des méthodes de choix pour l'exploration des moyens de prévention des CPM. Ainsi l'administration d'une levure d'intérêt médical - Saccharomyces bou- lardii - permet de réduire de $51 \%$ la mortalité induite par la clindamycine chez le hamster [20]. Ces observations ont trouvé des applications thérapeutiques chez l'homme, chez lequel cette levure a prouvé son efficacité en prévenant à la fois les diarrhées post-antibiotiques chez les patients porteurs de $C$. difficile et les rechutes de CPM traitées [21].

Récemment, Torres et al. [22] ont montré que l'administration orale de $100 \mu \mathrm{g}$ de toxine $\mathrm{A}$ à des rats induisait la synthèse d'un facteur antisécrétoire spécifique, comme cela a déjà été décrit pour Vibrio cholerae, Campylobacter jejuni et Escherichia coli. Ce facteur, synthétisé dans le système nerveux central à partir de la glande pituitaire et déversé dans la circulation sanguine, protégerait la muqueuse des effets cytotoxiques de la toxine A. Si son mécanisme d'action demeure encore obscur, on sait cependant qu'il n'affecte pas la fixation de la toxine $A$ à son récepteur.

\section{- Les animaux axéniques}

Bien que techniquement plus délicat à manipuler et financièrement plus onéreux, l'animal gnotoxénique* dont on peut contrôler la flore intestinale, constitue un modèle simplifié de choix pour l'étude des barrières microbiennes anti-Clostridium difficile et des facteurs pouvant moduler la production des toxines en évitant toutes les interférences liées à la flore normale complexe du tube digestif [18]. Le rat axénique chez lequel est introduit $C$. difficile est relativement résistant à l'effet létal de ce germe. En revanche, $17 \%$ des animaux qui meurent présentent des effets toxiques au niveau de différents organes: grêle, foie, poumons et surtout côlon où se développent - à la différence du hamster doré - des pseudomembranes adhérentes, similaires d'un point de vue histologique à celles observées chez l'homme [23]. La souris gnotoxénique chez laquelle est implanté $C$. difficile est un modèle animal particulièrement reproductible où la sévérité des symptômes est bien corrélée à la production de la to-

\footnotetext{
- Un animal gnotobiotique (ou gnotoxénique) est un animal axénique, c'est-à-dire sans germe, chez lequel on introduit un ou plusieurs germes connus.
} 
xine A [24] ; elle est très utilisée pour étudier l'influence des facteurs écologiques sur la production in vivo des toxines et la létalité. Ainsi, la nature protéique des régimes alimentaires (caséine) [25] et l'administration de certaines bactéries de la flore dominante de l'enfant (Escherichia coli, Streptococcus faecalis) ont déjà prouvé leur efficacité à améliorer la survie des souris chez lesquelles $C$. difficile a été introduit ; cet effet serait dû à une réduction sélective de la production de la toxine A [26]. De même, l'administration de Saccharomyces boulardii à des souris axéniques diminue significativement le taux de production de toxine A (celle de la toxine B est variable) sans affecter la colonisation du tube digestif des animaux par $C$. difficile [27]. Si les mécanismes cellulaires de ces différentes observations demeurent encore obscurs, leur intérêt en pathologie humaine est tout à fait évident.

\section{- Modèle de l'anse iléale ligaturée de lapin}

L'anse iléale de lapin représente un modèle particulièrement adapté à l'étude de l'effet local des toxines purifiées. De nombreux auteurs [28, 29] ont travaillé sur des lapins New Zealand mâles anesthésiés dont ils ont ligaturé l'iléon sur une portion de $10 \mathrm{~cm}$, afin de former une boucle dans laquelle ils peuvent injecter les toxines sous de faibles volumes; après canulation des pôles proximal et distal de l'anse ainsi formée, ils injectent un liquide de perfusion qu'ils recueillent de façon séquentielle en vue de l'analyse biochimique des sécrétions. Cette étude est complétée, d'une part, par l'évaluation de la perméabilité membranaire (mesure de la clairance sang-lumière intestinale du mannitol tritié) et, d'autre part, par une étude histologique des tissus lésés. Les résultats publiés par les différents auteurs sont concordants.

L'injection d'une faible quantité de toxine A $(25 \mu \mathrm{g})$ au niveau d'une anse ligaturée entraîne une sécrétion épaisse, purulente et hémorragique riche en débris cellulaires, mucus et protéines ; cette réponse dépend de la dose et de la durée. Elle est accompagnée d'une augmentation de la clairance au mannitol tritié, et d'une réponse inflammatoire intense objectivée par la libération de prostaglandines E2 et de leucotriènes B4. La muqueuse iléale est le siège d'importantes altérations, notamment une infiltration de polynucléaires plus dense au sommet des villosités que dans l'épithélium. L'injection de $100 \mu \mathrm{g}$ de toxine B n'induit aucune modification significative, mais potentialise l'action de la toxine A. Enfin, la même expérience réalisée sur des portions de côlon ligaturé n'entraîne aucun changement, tant sur le plan biochimique qu'histologique.

Les effets produits par la toxine A
Tableau II

COMPARAISON DES EFFETS DE LA TOXINE A DE $C$. DIFFICILE ET DE LA TOXINE CHOLÉRIQUE SUR LE MODĖLE DE L'ANSE ILÉALE LIGATURÉE DE LAPIN

\begin{tabular}{|l|c|c|}
\hline & Toxine A & Toxine cholérique \\
\hline $\begin{array}{l}\text { Sécrétion liquidienne } \\
\text { Nature de la sécrétion }\end{array}$ & $\begin{array}{c}+ \\
\text { inflammatoire } \\
\text { (PNN, GR, mucus) }\end{array}$ & $\begin{array}{c}++ \\
\text { aqueuse } \\
\text { (NaCI) }\end{array}$ \\
$\begin{array}{l}\text { Perméabilité } \\
\text { membranaire }\end{array}$ & augmentée & augmentée \\
Libération de PGE2 & oui & oui \\
Libération de LTB4 & oui & non \\
Adénylate cyclase & non stimulée & stimulée \\
Récepteur & glycoprotéine & glycolipide \\
\hline
\end{tabular}

(D'après Pothoulakis et al., 1990.)

PNN : polynucléaires neutrophiles; GR : globules rouges.

$\mathrm{m} / \mathrm{s} n^{\circ} 3$, vol. 8 , mars 92 sont différents en tout point de ceux engendrés par la toxine cholérique ; en effet, cette dernière, après s'être fixée sur un récepteur glycolipidique (ganglioside GM1), stimule une activité adénylate cyclase responsable d'un transsudat composé essentiellement d'eau et de sel (Tableau II).

Des expériences identiques peuvent être réalisées, non plus in vivo, mais ex vivo sur des explants d'iléon déposés dans un milieu de survie [29, $30]$; il est surprenant de constater l'absence de modification morphologique des villosités intestinales et d'altération de la perméabilité épithéliale. Ainsi, la nette contradiction entre les effets observés in vivo et ex vitro suggère bien l'existence d'un mécanisme d'action indirect : la plupart des auteurs s'accordent à penser que la toxine $\mathrm{A}$, après s'être fixée sur le récepteur de la bordure en brosse, exercerait une puissante action chimiotactique vis-à-vis des polynucléaires et cellules macrophagiques. Celles-ci seraient à l'origine d'une réponse inflammatoire intense, génératrice d'une destruction tissulaire voire d'une nécrose hémorragique. La toxine B "profiterait " de l'augmentation de perméabilité membranaire pour pénétrer plus profondément dans les différentes couches tissulaires et pour exercer son action cytotoxique localement (figure 3, p. 220). La brèche ainsi formée dans l'épithélium digestif pourrait favoriser le risque de dissémination systémique des toxines [2].

\section{- Les études in vitro}

Les études in vitro sont fondées sur l'observation des modifications cytologiques et biochimiques de différentes lignées cellulaires en culture soumises à l'action des toxines purifiées ; ces études ont permis d'éclaircir les mécanismes d'action cellulaire des toxines et confortent les hypothèses suggérées par les observations in vivo.

La toxine B est une puissante cytotoxine à laquelle de nombreuses lignées cellulaires sont sensibles : cellules fibroblastiques humaines ou animales (WI38, MRC5, L929), cellules rénales (BHK21, AGMK, Vero) ou autres cellules de lignées continues (CHO, HeLa, McCoy, HTC). Dans tous les cas, après action de la 


\section{RÉFÉRENCES}

24. Vernet A, Corthier G, Dubos-Ramare F, Parodi AL. Relationship between levels of Clostridium difficile toxin $\mathrm{A}$ and $\mathrm{B}$ and cecal lesions in gnotobiotic mice. Infect Immun $1989 ; 57: 2123-7$

25. Dubos-Ramare F, Corthier G. Influence of dietary proteins on production of Clostridium difficile toxins in gnotobiotic mice. Microb Ecology in Health and Disease $1990 ; 3$ : 231-4.

26. Corthier G, Dubos F, Raibaud P. Modulation of cytotoxin production by Clostridium difficile in the intestinal tracts of gnotobiotic mice inoculated with various human intestinal bacteria. App Environ Microbiol $1985 ; 49: 250-2$.

27. Corthier G, Dubos F, Ducluzeau R. Prevention of Clostridium difficile induced mortality in gnotobiotic mice by Saccharomyces boulardii. Can J Microbiol 1986; 32 : 894-6.

28. Triadafilopoulos G, Pothoulakis C, Weiss R, Giampaolo C, LaMont JT. Comparative study of Clostridium difficile toxin A and cholera toxin in rabbit ileum. Gastroenterology $1989 ; 97$ : 1186-92.

29. Lima AAM, Lyerly DM, Wilkins TD, Innes DJ, Guerrant RL. Effects of Clostridium difficile toxins $\mathrm{A}$ and $\mathrm{B}$ in rabbits small and large intestine in vivo and on cultured cells in vitro. Infect Immun $1988 ; 86$ : 582-8.

30. Triadafilopoulos G, Pothoulakis C, O'Brien MJ, LaMont JT. Differential effects of Clostridium difficile toxins A and B on rabbit ileum. Gastroenterology 1987 ; 93 : 273-9.

31. Maniar AT, Trevor WW, Hammond GW. Detection of Clostridium difficile toxin in various tissue culture monolayers. J Clin Microbiol 1987 ; 25 : 1999-2000.

32. Mitchell MJ, Laughon BE, Lin S. Biochemical studies on the effect of Clostridium difficile toxin $\mathrm{B}$ on actin in vivo and in vitro. Infect Immun 1987; 55:1610-5

33. Rihn B, Beck G, Monteil H, Lecerf F, Girardot R. Effect of cytotoxin of Clostridium difficile on cultured hepatoma cells. Biol Cell $1985 ; 53: 23-32$.

34. Tucker KD, Carrig PE, Wilkins TD. Toxin A of Clostridium difficile is a potent cytotoxin. Infect Immun $1990 ; 28$ : 869-71.

35. Fiorentini C, Malorni W, Paradisi S, Giuliano M, Mastrantonio P, Donelli G. Interaction of Clostridium difficile toxin A with cultured cells : cytosqucletal changes and nuclear polarization. Infect Immun $1990 ; 58$ : 2329-36

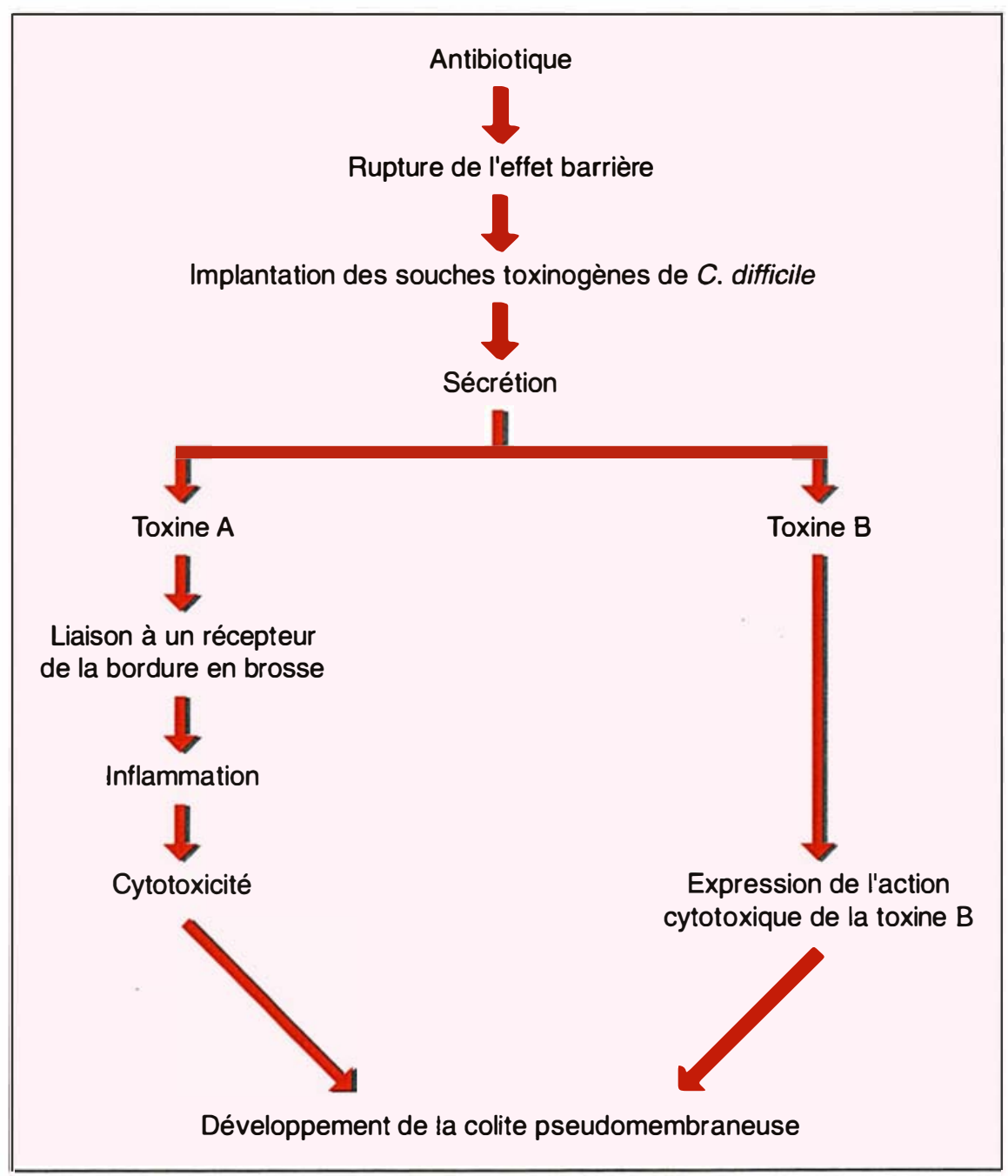

Figure 3. Mécanisme d'action hypothétique des toxines de C. difficile.

toxine B, les cellules s'arrondissent et prennent un aspect actinomorphique* $^{*}$; la recherche d'un tel effet cytopathogène après inoculation d'un filtrat de selles stérile est à la base du diagnostic biologique des infections digestives à $C$. difficile [31]. Les modifications morphologiques induites par l'inoculation de $20 \mathrm{ng}$ de toxine B sur des cultures de cellules fibroblastiques humaines $3 \mathrm{~T} 3$ sont similaires à celles produites par la

- Aspect actinomor phique : aspect d'arborisation des cellules lié à un effondrement du cytoplasme autour du noyau, occasionné par un raccourcissement des filaments d'actine. cytochalasine $\mathrm{B}$, molécule qui dépolymérise l'actine $F$ [32] : elles consistent en un effondrement du cytoplasme autour du noyau conférant à la nappe cellulaire un aspect d'arborisation ; cet effet cytopathogène semble être précédé d'une réorganisation des filaments d'actine qui diminuent, se raccourcissent et se condensent autour du noyau alors que le pool d'actine monomérique augmente ; néanmoins, l'absence d'effet de la toxine B sur des préparations d'actine purifiée in vitro suggère que son mécanisme d'action est indirect, s'effectuant sans doute par le biais des nombreuses protéines du cytosquelette associées à l'actine (vinculine, gelsoline, filamine, etc.). 
D'un point de vue biochimique, de nombreux auteurs ont montré que les modifications morphologiques s'accompagnent de perturbations biochimiques : perte de potassium intracellulaire, diminution de la synthèse des protéines, des acides ribonucléiques et désoxyribonucléiques [33].

Comme la toxine $\mathrm{B}$, la toxine A est cytotoxique, mais son action est $10^{3}$ à $10^{4}$ fois inférieure à celle de la toxine B. Néanmoins, contrairement à cette dernière, toutes les variétés cellulaires ne sont pas également sensibles à l'action de la toxine $\mathrm{A}$ : en effet, les cellules d'explants primaires et les cellules intestinales y sont insensibles, alors que celles issues de tératocarcinome P19 ou F19 ou les cellules $\mathrm{CaCO}_{2}$ y sont extrêmement sensibles : cette différence serait liée à la présence d'un plus ou moins grand nombre de récepteurs de la toxine $\mathrm{A}$ au niveau de la surface des cellules [34].

Si les modifications morphologiques et biochimiques induites par la toxine $\mathrm{A}$ sur des lignées sensibles sont semblables à celles induites par la toxine $\mathrm{B}$, elles ne sont cependant pas identiques. Les modifications morphologiques de cellules HeLa exposées à la toxine A surviennent en trois temps [35]: on peut observer tout d'abord une rétraction cellulaire avec modification de l'adhésivité mais conservation de l'intégrité du cytosquelette ; puis, dans un deuxième temps, apparaît un arrondissement cellulaire lié à une concentration de l'actine $\mathrm{F}$ et de l' $\alpha$-actinine au pôle opposé au noyau tandis que des modifications encore mal définies du système microtubulaire et des filaments intermédiaires entraînent une polarisation nucléaire ; enfin, la dernière étape consiste en une nécrose cellulaire accompagnée d'une désorganisation totale du cytosquelette.

Hecht et al. [36] ont montré que les altérations du cytosquelette des cellules T84 dérivées d'un cancer humain de côlon étaient concomitantes des modifications de perméabilité cellulaire. En effet, les courants ioniques de ces cellules en monocouche sont contrôlés par des jonctions de type " serré ". Lorsque ces cultures cellulaires sont exposées à la toxine $\mathrm{A}$ $(0,7 \mu \mathrm{g} / \mathrm{ml})$ purifiée, la résistance transépithéliale disparaît en 6 à $\mathrm{m} / \mathrm{s} n^{\circ} 3$, vol. 8 , mars 92
8 heures. Cette diminution considérable de la résistance électrique est attribuée à une disparition des filaments d'actine au niveau des anneaux périjonctionnels, permettant alors un passage du mannitol par des chemins paracellulaires.

La forte activité chimiotactique de la toxine $\mathrm{A}$ vis-à-vis des polynucléaires et l'augmentation rapide des concentrations de calcium intracellulaire qu'elle induit dans ces cellules ont été mises en évidence par Becker et al. [37] à partir de culture de cellules basophiles de souris leucémiques. Ces cellules possèdent à leur surface un récepteur pour la toxine $\mathrm{A}$ proche de celui de la bordure en brosse, couplé à une GTP-protéine. Les études entreprises par la suite sur des cultures mixtes de macrophages et de lymphocytes spléniques de souris suggèrent que la toxine $A$ stimulerait la libération des cytokines (notamment l'interleukine 1) par les macrophages qui, en retour, permettraient une activation des lymphocytes. Selon Miller et al. [38], la toxine A pourrait bien, in vivo stimuler les cellules macrophagiques de la lamina propria, entraînant ainsi une libération de cytokines et de médiateurs de l'inflammation qui, à leur tour, favoriseraient l'afflux des polynucléaires dans la lamina propria. Une autre hypothèse propose que la toxine A agirait directement sur l'entérocyte qui synthétiserait une molécule chimiotactique responsable d'une réaction inflammatoire locale.

\section{Autres facteurs de virulence}

Si la toxine A et la toxine B constituent bien les deux principaux facteurs de pathogénicité de $C$. difficile, d'autres molécules toxiques ont été caractérisées : leur mise en évidence et leur rôle exact devront être confïmés par différentes équipes indépendantes.

Parmi celles-ci, une entérotoxine non hémorragique, distincte de la toxine $\mathrm{A}$, a été isolée par Banno et al. [39].

Torres et al. [40, 41] ont isolé, purifié et caractérisé trois nouvelles protéines cytotoxiques (regroupées sous le nom de fraction $\mathrm{C}$ ), différentes d'un point de vue biochimique, antigénique et biologique des toxines $\mathrm{A}$ et $\mathrm{B}$.
La fraction $\mathrm{C}$ serait responsable d'une détérioration modérée de la partie apicale des villosités intestinales sans atteinte des cryptes, et d'une sécrétion fluide. La fraction $\mathrm{C}$ serait dépourvue d'effet sur la muqueuse colique. Ces auteurs suggèrent que l'expression complexe des interactions entre ces différentes toxines produites par $C$. difficile pourraient expliquer le large spectre clinique (diarrhées, colites, CPM) associé à cette bactérie. Une protéine de haut poids moléculaire a également été mise en évidence : elle agirait en modifiant le potentiel électrique de segments isolés d'intestin de lapin mais n'aurait aucune activité entérotoxique ou cytotoxique [42].

Popoff et al. [43] ont isolé une souche de $C$. difficile qui possède une activité ADP-ribosyltransférase spécifique de l'actine, proche de celle de la chaîne légère de la toxine $\mathrm{C} 2$ de Clostridium botulinum.

Certaines souches de $C$. difficile produisent des enzymes hydrolytiques : hyaluronidases, chondroïtine sulfatases, collagénases, héparinases, neuraminidases, gélatinases. Cependant, aucune relation significative n'a pu être formellement démontrée entre la production de ces composés et la virulence des souches [44].

Outre les facteurs toxiques, les propriétés d'adhèrence de $C$. difficile aux cellules hôtes semblent constituer des facteurs essentiels de l'expression de la virulence. En effet, Borriello et al. [38] ont montré que les souches hautement virulentes adhèrent significativement mieux que les souches non virulentes à la muqueuse intestinale du hamster prétraité par la clindamycine : les mécanismes intimes de cette adhérence demeurent encore obscurs en dépit des observations réalisées sur des modèles in vivo et in vitro. Les mécanismes d'adhérence sont encore hypothétiques : fixation non spécifique par des fimbriae présents sur certaines souches de $C$. difficile ou par l'intermédiaire de la toxine ou par des liaisons hydrophobes, ou bien fixation spécifique de type ligand-récepteur, etc.

\section{Conclusion}

Une dizaine d'années se sont écoulées depuis la découverte de $C$. difficile comme l'agent responsable de 


\section{RÉFÉRENCES}

36. Hecht G, Pothoulakis C, LaMont JT, Madara JL. Clostridium difficile toxin A perturbs cytosqueletal structure and tight junction permeability on cultured human intestinal epithelial monolayers. J Clin Invest 1988 ; 82 : 1616-24.

37. Becker SD, Pothoulakis C, Dickey B, LaMont JT. Clostridium difficile toxin A binds to a G-protein coupled receptor on rabbit neutrophil membranes. Gastroenterology 1989 ; 96 : A36 (abstract).

38. Miller PD, Pothoulakis C, Becker TR, LaMont JT, Rothstein TL. Macrophagedependent stimulation of $\mathrm{T}$ cell depleted spleen cells by Clostridium difficile toxin A and calcium ionophore. Cell Immun 1990 ; 126 : 155-63.

39. Banno $\mathrm{Y}$, Kobayashi $\mathrm{T}$, Kono $\mathrm{H}$, Watanabe K, Ueno K, Nozawa Y. Biochemical characterization and biologic actions of two toxins (D1 and D2) from Clostridium difficile. Rev Infect Dis 1984; 6 (suppl 1) : S11-20.

40. Torres JF, Lönnroth I. Production, purification and characterization of Clostridium difficile toxic proteins different from toxin A and toxin B. Biochim Biophys Acta $1989 ; 998: 151-7$.

41. Torres JF, Jennische E, Lange S, Lönnroth I. Enterotoxins from Clostridium difficile; diarrhoeogenic potency and morphological effects in the rat intestine. Gut 1990 ; 31 : 781-5.

42. Justus PG, Martin JL, Goldberg DA, et al. Myoclectric effects of Clostridium difficile : motility altering factors distinct from its cytotoxin and enterotoxin in rabbits. Gastroenterology $1982 ; 83: 836-43$.

43. Popoff MR, Rubin EJ, Gill DM, Boquet $P$. Actin-specific ADP-ribosyltransferase produced by a Clostridium difficile strain. Infect Immun 1988 ; 56 : 2299-306.

44. Borriello SP, Davies HA, Kamiya S, Reed PJ, Seddon S. Virulence factors of Clostridium difficile. Rev Infect Dis $1990 ; 12$ (suppl 2) : 185-91.

45. Pothoulakis C, Becker SD, LaMont JT. Mechanism of action of Clostridium difficile toxins. In : Rambaud JC, Ducluzeau R, eds. Clostridium difficile et pathologie intestinale. Paris : Springer-Verlag, 1990: 55-61.
CPM et de diarrhées postantibiotiques. Des progrès considérables ont été réalisés tant sur le plan de la connaissance physico-chimique des toxines $\mathrm{A}$ et $\mathrm{B}$ que de leurs mécanismes d'action: la toxine $\mathrm{A}$, qui serait le principal facteur de pathogénicité, se fixerait au niveau d'un récepteur situé sur la membrane de la bordure en brosse et aurait deux types d'action. D'une part, elle agirait directement au niveau cellulaire par dépolymérisation des filaments d'actine de cellules entérocytaires, responsable d'une altération du cytosquelette et d'une augmentation de perméabilité membranaire. D'autre part, elle induirait de manière indirecte une réponse inflammatoire intense au sein de la lamina propria : les médiateurs de l'inflammation (histamine, leucotriènes, prostaglandines, interleukines) ainsi libérés contribueraient à la formation de lésions épithéliales, à l'augmentation de la perméabilité membranaire, à la sécrétion d'un liquide mucopurulent. La muqueuse intestinale ainsi endommagée permettrait alors à la toxine $\mathrm{B}$ - incapable d'agir seule sur la muqueuse - d'exercer sa puissante activité cytotoxique et ainsi de potentialiser les effets de la toxine A. Néanmoins, l'extrapolation à l'homme doit être prudente, tant il est clair que les différentes espèces animales peuvent présenter des différences de sensibilité à la même souche de $C$. difficile.

Malgré les récents progrès accomplis dans la connaissance de la physiopathologie des infections digestives à $C$. difficile, de nombreuses questions restent en suspens : pourquoi les nouveau-nés hébergent-ils fréquemment des souches toxinogènes tout en étant réfractaires à la CPM ? Comment peut-on expliquer que la prolifération de $C$. difficile au sein de l'écosystème microbien du côlon puisse être induite par des antibiotiques auxquels cette bactérie est sensible? Quels sont les mécanismes potentiels permettant d'expliquer l'effet barrière ?

\section{TIRÉS A PART}

J.-C. Petit.

\section{Summary}

Pathophysiology of Clostridium difficile-associated intestinal diseases

Clostridium difficile (CD) is the main causative agent of pseudomembranous colitis in humans and is also strongly involved in antibioticassociated diarrhea. Toxigenic strains of this anaerobic bacterium produce two large, heat labile and cytotoxic proteins that are lethal in animals : a toxin A (enterotoxin) and a toxin B (cytotoxin). These toxins have been largely studied in the past 5 years, both in vivo (in the hamster model, in gnotobiotic mice, in the rabbit ileal loops) and in vitro (in cultured cells). The initial step of the disease seems to involve the disruption of the natural intestinal flora caused by antibiotics, the CD overgrowth and the binding of toxin A on a specific glycoprotein receptor in the brush border membrane. Then, by a signal process which is not yet fully understood, toxin A elicits infiltration of neutrophils in the lamina propria. Release of inflammatory mediators from macrophages and mast cells leads to an hemorragic necrosis, a fluid secretion, an increase of cell permeability, and an alteration of smooth muscle functions. The extensive damage which occurs from the action of toxin $\mathrm{A}$ and the intense inflammatory infiltrates result in a dissemination of both toxin A and B into the systemic circulation. Toxin B has no direct effect on the intestinal epithelium but seems to act in synergy with toxin A and displays a strong cytopathic activity. Other virulence factors seem to play a role (adhesion properties, secretion of hydrolytic enzymes, production of a capsule...) but additional studies are required to learn more about their relevance. 